This is an author produced version of a paper published in Atherosclerosis. This paper has been peer-reviewed but does not include the final publisher proof-corrections or journal pagination.

Citation for the published paper:

Skoog, Maria and $\mathrm{Xu}$, Ning and Berggren-Soderlund, Maria and Lovegrove, Julie A and Nilsson-Ehle, Peter.

"ACTH reduces the rise in ApoB-48 levels after fat intake. "

Atherosclerosis, 2006, Issue: July 11.

http://dx.doi.org/10.1016/j.atherosclerosis.2006.05.012

Access to the published version may require journal subscription. Published with permission from: Elsevier 


\section{ACTH reduces the rise in ApoB-48 levels after fat intake}

Maria Skoog†, Ning Xu†, Maria Berggren-Söderlund†, Julie A. Lovegroveł and Peter Nilsson-Ehle†§.

† Division of Clinical Chemistry and Pharmacology, Department of Laboratory medicine, Lunds University, S-221 85 Lund, Sweden

$\ddagger$ Hugh Sinclair Unit of Human Nutrition, School of Food Biosciences, University of Reading, Reading, Berks RG6 6AP, United Kingdom

Key words: ACTH, apoB, lipoprotein metabolism, postprandial

Number of figures: 3

Number of Tables: 2

$\S$ Address correspondence:

Peter Nilsson-Ehle

Division of Clinical Chemistry and Pharmacology,

ILM, Lunds University

S-221 85 Lund, Sweden

Telephone: +46 46 173452, Fax: 4646130064

E-mail: peter.nilsson-ehle@med.lu.se 


\begin{abstract}
It has been repeatedly demonstrated that ACTH administration lowers plasma lipid concentrations in man. The present study was designed to test the hypothesis, based on observations of decreased apolipoprotein B (ApoB) synthesis and secretion in vitro, that ACTH administration inhibits the postprandial output of ApoB in man. Therefore, we studied the response to a fat-rich meal supplemented with vitamin A in eight healthy volunteers, who underwent this test without premedication, after 4 day's administration of ACTH, and after 4 day’s administration of a glucocorticoid (betamethasone).

As expected, fasting plasma levels of low-density lipoproteins (LDL)-cholesterol (-25\%) and apoB (-17 \%) decreased after ACTH, but not after betamethasone administration. Also, the elevation of plasma ApoB-48 in response to fat intake (to twice the basal levels) was markedly reduced after ACTH administration. However, the postprandial rise in plasma triglycerides and retinyl palmitate was unimpaired, suggesting that ACTH administration induced the secretion of fewer but larger chylomicrons. The effect of betamethasone on the postprandial response was similar but less pronounced.

This study confirms earlier reports on the lipid-lowering effects of ACTH and supports our theory, based on in vitro studies, that the lipid-lowering effects of ACTH administration in man involves an inhibition of ApoB production.
\end{abstract}




\section{Introduction}

It is well known that ACTH regulates important aspects of lipid metabolism in the adrenal glands, primarily the uptake of cholesterol for synthesis of steroid hormones $(1,2)$. It has also been demonstrated that administration of exogenous ACTH in man has beneficial effects on lipid metabolism. A pronounced and consistent decrease of plasma cholesterol and lowdensity lipoproteins (LDL) -cholesterol by 20- 40\% has been reported during ACTH treatment in healthy individuals $(3,4)$, in steroid-treated patients with renal disease $(5,6)$ and in hemodialysis patients $(4,7)$. The hypolipidemic effect includes all apolipoprotein B (ApoB) -containing lipoproteins, i.e. very low-density lipoproteins (VLDL), LDL and Lipoprotein (a). These effects can be attributed to ACTH as such, since they are clearly distinct from the effects of equipotent doses of glucocorticoids (3).

The mechanisms behind the lipid-lowering actions of ACTH, however, are not yet elucidated. Theoretically, ACTH may affect production as well as elimination of lipoproteins. There is support for both alternatives. Although ACTH does not seem to modulate the activity or expression of the LDL receptor or scavenger receptor-BI (8) there is evidence that increased removal rate may be one mechanism of action. Thus, we have suggested, from observations in clinical studies, that ACTH may act by enrichment of VLDL and LDL with apoE $(4,7)$. Due to its high affinity to lipoprotein receptors, apoE would mediate an accelerated lipoprotein removal from the circulation $(4,7)$. On the other hand, studies in vitro suggest that the mechanism behind the cholesterol-lowering effect of ACTH is also related to decreased synthesis of ApoB-containing lipoproteins. Thus, ACTH significantly and selectively suppresses ApoB mRNA levels and ApoB secretion in the human hepatoma cell line HepG2 (8). 
To explore whether decreased ApoB production and/or secretion, as noted in vitro, is also involved in the lipid-lowering effects of ACTH in vivo, we used the enterocyte as a readily accessible model to study synthesis and assembly of ApoB containing lipoproteins in man. The production of ApoB-48 and assembly of chylomicrons in the intestine (9) resembles that of ApoB-100 production and VLDL synthesis in the liver (10). In the present investigation we monitored the effects of ACTH on the formation of ApoB-48 in response to dietary intake of fat, with the hypothesis that ACTH administration in vivo would blunt or abolish the postprandial rise in plasma apoB concentrations. In the design of the experiment, we anticipated that the early phase $(0-4 \mathrm{~h})$ of the postprandial response would be particularly informative with regard to intestinal lipoprotein formation and secretion (11), whereas the later phase (4-7 h) would increasingly reflect intravascular metabolism and elimination of lipoproteins (12). 


\section{Subjects and Methods}

2.1 Subjects and experimental design. Healthy volunteers, five men and five women, were recruited for participation. Their ages were for men 18-42 years (median 33) and for women 25-51 years (median 34). Medical history, physical examination and a set of routine tests did not reveal signs of liver or kidney dysfunction or metabolic diseases. The studies were approved by the ethics' committee at Lund University and the volunteers gave their informed consent to participation.

In each participant we studied responses to an oral fat load with no premedication (basal) and after ACTH and betamethasone administrations. Hormones were administered on four consecutive days, and on the fifth day the oral fat load was carried out. There was a six-month interval between the two administrations. Two participants (1 man and 1 woman) could not participate during the betamethasone study.

2.2 Drug administration. ACTH was given as tetracosactid, $\mathrm{ACTH}_{1-24}$, (Synacthen Depot, Novartis Sverige AB, Nycomed Austria GmbH, Linz, Austria), at a dose of $1 \mathrm{mg} / \mathrm{day}$ as an intra-muscular injection in the morning. Glucocorticoids were administered orally as the synthetic betamethasone (Betapred, Defiante Farmacëutica Unipessoal, Sigma-Tau, Pomezia, Italy), at a dose of $2 \mathrm{mg}$ three times daily.

2.3 Oral fat load. After an overnight fast the participants ate a test meal containing $120 \mathrm{~g}$ of fat and ingested a vitamin-A tablet containing $50000 \mathrm{IU}$ of retinyl palmitate (Arovit, Roche AB, F.Hoffmann-La Roche Ltd, Basel, Switzerland). The meal was consumed within twenty minutes and the participants were fasting for the following seven hours, but had free access to 
water. Total energy of the meal was 6266 kJoule provided by fat (71\%), carbohydrates (19\%) and proteins (10\%).

2.4 Blood sampling and laboratory analyses. Participants received an intravenous catheter, and blood samples were drawn in the morning after an overnight fast. Postprandial blood samples were then obtained repeatedly during 7 hours. Serum tubes were protected against light to prevent degradation of retinyl palmitate. Blood samples were either analysed immediately, at the routine laboratory of clinical chemistry at Lund University Hospital, or separated and stored for later analysis in the same series.

Routine analyses were performed by accredited methods for P-Cholesterol, P-Triglycerides, P-high-density lipoprotein (HDL)-cholesterol, P-LDL-cholesterol, P-ApoB, P-ApoA-I, PGlucose and P-Cortisol. S-Phospholipids were analysed with a colorimetric kit (Phospholipids B, Boule Nordic AB, Wako Chemicals GmbH, Neuss, Germany). S-Retinyl palmitate was analysed by high-performance liquid chromatography (HPLC) essentially as described by Nierenberg and Lester (13) with the following adjustments to apply to retinyl palmitate: the internal standard was retinyl acetate $(0,5 \mu \mathrm{g} / \mathrm{ml})$,the detection wavelength was $325 \mathrm{~nm}$, the mobile phase was methanol and Nucleosil C18 $(5 \mu \mathrm{m}, 200 * 4,6 \mathrm{~mm})$ was used at a temperature of $45^{\circ} \mathrm{C}$. P-ApoB- 48 was analysed using a competitive enzyme-linked immunosorbant assay (ELISA) method (14) with minor modifications. Briefly, a heptapeptide-thyroglobulin conjugate consisting of the C-terminal residues of ApoB-48 was used as coating material. Samples were diluted 1:5 with PBS, incubated with a specific polyclonal anti-ApoB-48 antibody that recognizes the C-terminal region of ApoB-48 and does not cross-react with ApoB-100 (15). A standard curve was prepared by serial dilution of the ApoB-48 heptapeptide in phosphate buffer saline (PBS) containing $10 \mathrm{~g} / \mathrm{l}$ human serum albumin. Both 
inter- and intra-individual variations in P-ApoB-48 were similar to those found previously although no preservatory agent was used in the current study.

2.5 Statistical Analyses. Data were analysed with a nonparametric, 2-tailed Wilcoxons paired signed rank test using Graph Pad Prism software, version 3.0c. Alterations in P-ApoB-48 levels during the early postprandial phase (0-4 h) were estimated as area under the curve (AUC) by the trapezoid rule and analysed with analysis of variance (ANOVA) and when appropriate Wilcoxons paired signed rank test in Graph Pad Prism software. 


\section{Results}

Both hormone administrations were well tolerated by most participants. A few signs of increased mental activity, irritability and fluid retention were noted towards the end of the administrations, all well known effects of the administered hormones. Since concentrations of immunoglobulins and albumin in plasma are unchanged during these hormone administrations $(3,6)$, the observed alterations in measured plasma/serum constituents are not attributed to changes in plasma volume.

3.1 Cortisol and glucose. Administration of ACTH markedly increased plasma cortisol concentrations, while betamethasone significantly suppressed plasma cortisol levels (Table 1). Both ACTH and betamethasone administrations led to significantly elevated fasting plasma glucose levels (Table 1), which were maintained throughout the postprandial period (data not shown). This indicates that the two hormone treatments were physiologically active with typical metabolic effects, and that the doses of ACTH and betamethasone had equipotent glucocorticoid effects.

3.2 Lipids and apolipoproteins in the fasting state. As expected, four days' administration of ACTH resulted in reductions in plasma concentrations of the major components of LDL and VLDL (Table 2). Thus, median plasma levels of cholesterol decreased by 13\%, phospholipids by $17 \%$, LDL-cholesterol by $25 \%$ and ApoB-100 by $17 \%$. Due to the wide intra-individual variation in triglyceride concentrations, the reduction in P-Triglyceride did not reach statistical significance. In contrast, betamethasone did not affect any of these variables significantly. 
HDL was differently affected by the two hormonal regimens (Table 2). ACTH induced a moderate decrease in the level of ApoA-I, while betamethasone administration was associated with a rise in HDL-cholesterol, indicating a slight decrease in $\mathrm{HDL}_{3}$ after $\mathrm{ACTH}$ and a moderate increase in the (cholesterol-rich) $\mathrm{HDL}_{2}$ subfraction after betamethasone, as demonstrated previously (3).

As expected the concentrations of ApoB-48 in the fasting state were low, but were significantly elevated after ACTH as well as after betamethasone administration (Table 2).

3.3 Postprandial effects. As expected, LDL and HDL were not affected by food intake, as evidenced by the constant plasma concentrations of cholesterol, phospholipids, LDLcholesterol, ApoB, HDL-cholesterol and ApoA-I during the postprandial period (data not shown).

3.3.1 ApoB-48. In response to the oral fat load plasma ApoB-48 levels increased rapidly and reached a maximum after four hours (Figure 1.a). After ACTH administration the postprandial rise in P-ApoB-48 was markedly impaired, most clearly during the early phase (0-4 h) of the postprandial response. The response to betamethasone was similar but less pronounced. The integrated change in P-ApoB-48 during the early postprandial phase, expressed as AUC (Figure 1.b), was significantly smaller after ACTH as well as betamethasone, indicating a reduced postprandial ApoB-48 output after hormone administrations.

3.3.2 Dietary Markers. Plasma triglyceride concentrations increased postprandially and reached a maximum after four hours (Figure 2). In contrast to the postprandial response of 
ApoB-48, there was no impairment of the triglyceride absorption in response to hormone administrations (n.s, Anova).

Serum retinyl palmitate levels also increased postprandially (Figure 3). As observed for plasma triglyceride concentrations, the postprandial retinyl palmitate levels were not impaired in response to the hormone administrations; rather, retinyl palmitate levels after both hormone administrations in the early postprandial phase were elevated $(\mathrm{p}<0,05)$. 


\section{Discussion}

This study further confirms earlier reports on the lipid-lowering effects of ACTH and supports our theory, based on in vitro data, that the lipid lowering effects of ACTH administration in man involves an inhibition of the synthesis of ApoB (8). Thus, the action of ACTH would be at least in part attributed to a reduced production and secretion of apoB-containing lipoproteins.

Consistent with earlier reports $(3-7,16,17)$, plasma concentrations of major constituents of VLDL/LDL (ApoB, LDL-cholesterol, triglycerides and phospholipids) in the fasting state were markedly reduced in response to ACTH administration. The hypolipidemic effect of ACTH was essentially restricted to VLDL/LDL, and the effects on HDL components were much less pronounced. The effects on VLDL/LDL constituents were due to ACTH as such, since administration of betamethasone, a potent glucocorticoid, led to moderately increased plasma concentrations of ApoB, LDL-cholesterol, triglycerides and phospholipids (3). The minor divergences with regard to HDL components and triglycerides, in this and earlier studies $(3-7,16)$ may be attributed to the choice of study population (healthy subjects, patients with renal disease and various degrees of hyperlipidemia, predominantly men or women).

A novel, and surprising, finding was a moderate increase in plasma ApoB-48 concentrations in the fasting state after ACTH administration, most likely due to a glucocorticoid effect. It may reflect a retention of largely lipolysed “chylomicrons” in the circulation, an interpretation consistent with the significant reduction of hepatic lipase activity during ACTH administration reported in earlier studies $(3,17)$. Hepatic lipase is involved in the late stages of the intravascular delipidation of triglyceride-rich lipoproteins (18-20), and an impairment of the final steps in the lipolytic process might conceivably retard the transformation to chylomicron remnants with full affinity for its receptors. 
To test the hypothesis that decreased synthesis of ApoB, as observed in vitro (8), is involved in the reduction of ApoB-containing lipoproteins in response to ACTH in vivo, this study was designed to monitor the effects of ACTH on plasma concentrations of ApoB-containing lipoproteins, which rapidly increase after fat intake. This postprandial lipidemia is mainly accounted for by intestinal production of chylomicrons, which contain ApoB-48. Intestinal ApoB-48 is homologous to the N-terminal 2152 amino acids of hepatic ApoB-100 (21), due to their common origin in a single, human ApoB gene, $A P O B$ (22), and is formed through editing of full length ApoB mRNA occurring predominantly in the intestine in humans (23, 24). Both ApoBs are essential structure proteins for assembly and secretion of chylomicrons and VLDL, respectively. The secretory pathways for triglyceride-rich lipoproteins in the liver and intestine share several characteristics including postsecretory requirement for microsomal triglyceride-transfer protein (MTP), and both are sensitive to lipid availability $(9,10,25)$.

The postprandial phase is thus a readily accessible model for ApoB production and/or secretion. However, it should be borne in mind that the postprandial phase is a complex model which reflects several aspects of intestinal lipoprotein metabolism: during the early phase of the postprandial response, changes in plasma concentrations are mainly due to alterations in lipoprotein production and secretion, whereas intravascular metabolism (lipoprotein lipase and hepatic lipase) and elimination (LDL receptor, LRP) of these particles are increasingly important determinants of lipoprotein concentrations at later stages. In addition ACTH and glucocorticoids, being stress-related hormones, may affect the gastrointestinal motor complex (26), possibly via the hypothalamic-pituitary-adrenal axis (27, 28). Furthermore, inherent features of the experiment (prolonged fasting etc) may influence the variables under study. In some calculations we have therefore distinguished between information derived from the 
early (0-4 hours) and late part of the postprandial response. In this context it deserves to be pointed out that administration of ACTH leads to a significant reduction of hepatic lipase activity, while the activity of lipoprotein lipase in healthy subjects is unaffected by ACTH (3, 17).

In the present study, ACTH administration markedly reduced the rise in plasma ApoB-48 after a fat meal. Theoretically this can be due to enhanced clearance of chylomicron remnants e g through a decreased competition for clearance due to the decreased synthesis of VLDL/LDL seen after ACTH administration. However, the moderate increase of apoB-48 levels in the fasting (basal) state suggests that the clearance of apoB-48-containing remnants is not enhanced during ACTH administration. Thus, our results are consistent with the hypothesis that ACTH administration is associated with a reduced output of chylomicrons after a fat meal. This interpretation is in line with our previous in vitro study, where decreased intracellular levels of ApoB mRNA and decreased secretion of ApoB was seen in liver cells after exposure to ACTH in vitro (8). Taken together, these observations are consistent with the view that impaired synthesis of ApoB is also involved in the lipidlowering action of ACTH in man in vivo. The present study does not allow conclusions as to the detailed biological mechanisms; although modulation of ApoB gene transcript is probable, as previously demonstrated in HepG2 cells (8), presecretory degradation of ApoB, a wellestablished regulatory mechanism, is also possible. Further studies using human intestinal (Caco2) and hepatic (HepG2) cell lines may be useful tools to further delineate the inhibitory mechanisms of ACTH on ApoB synthesis.

Although the number of chylomicrons secreted into plasma (i.e. ApoB-48 concentrations) seemed essentially constant after ACTH administration (Figure 1a), absorption of dietary 
components (retinyl palmitate and triglycerides) was not affected in the same manner (Figure 2 and Figure 3). Rather, lipid transfer to the plasma compartment seemed accelerated, probably due to glucocorticoid effects on the GI motor complex $(27,28)$.This suggests that fewer but larger, i.e. fat-richer, chylomicrons are generated after ACTH administration (29, 30). In fact, the observation that dietary markers did not display similar postprandial plasma patterns as ApoB-48 is consistent with the view that the lipid-lowering effect of ACTH is due to direct inhibition of ApoB synthesis, since the decreased secretion of lipoproteins does not seem to result from decreased lipid availability.

Administration of ACTH had a more pronounced inhibitory effect on postprandial plasma ApoB-48 levels than glucocorticoid administration. This suggests that the two hormones act by different mechanisms to reduce ApoB-48 postprandially. Differences in the elimination of chylomicrons are less likely since the postprandial plasma levels of triglycerides and retinyl palmitate, and also the plasma levels of ApoB-48 in the fasting state after both hormone administrations were elevated. Thus, differences in ApoB production seem to be involved. The fact that betamethasone has no inhibitory effect on plasma ApoB-100 concentrations indicates that the effects of betamethasone on ApoB metabolism are limited to the processing of ApoB-48 in the intestine. The mRNA-editing in intestinal cells is an obvious possibility, although intestinal and hepatic cells also diverge to some extent regarding ApoB transcriptional factors (31). The catalytic deaminase, Apobec-1, and auxiliary proteins, the number and functions of which are yet not fully known, are involved in nuclear editing of ApoB mRNA in the enterocyte, resulting in ApoB-48 (32). In humans, editing is known to be regulated during embryogenesis (33), with the adult pattern of $>90 \%$ edited mRNA established in the third trimester, and in vitro models or in rodents several metabolic 
hormones such as insulin, dexamethasone, growth hormone, T4 and cortisol have been shown to modulate editing or mRNA levels (34-37).

In conclusion, ACTH administration in man strongly reduced the postprandial rise of ApoB48, while the postprandial plasma levels of dietary lipid components were unimpaired. Hormonal administration thus results in the secretion of fewer but larger chylomicrons. Our results support the hypothesis that decreased synthesis of ApoB is involved in the hypolipidemic effects of ACTH. 


\section{Acknowledgments}

We thank Siv Svensson, Kerstin Larsson, Gerd Nilsson and Sean Lovegrove for excellent technical assistance. Financial support was obtained from the Swedish Medical Research Council (04966), the Physiographic Society of Lund, the Albert Påhlsson Foundation, the Medical Faculty and Lund University Hospital. 


\section{References}

1. Alfano, J., Pedersen, R.C., Kramer, R.E., and Brownie, A.C. 1983. Cholesterol metabolism in the rat adrenal cortex: acute temporal changes following stress. Can J Biochem Cell Biol 61:708-713.

2. Boggaram, V., Funkenstein, B., Waterman, M.R., and Simpson, E.R. 1984. Lipoproteins and the regulation of adrenal steroidogenesis. Endocr Res 10:387-409.

3. Berg, A.L., and Nilsson-Ehle, P. 1994. Direct effects of corticotropin on plasma lipoprotein metabolism in man--studies in vivo and in vitro. Metabolism 43:90-97.

4. Arnadottir, M., Dallongeville, J., Nilsson-Ehle, P., and Berg, A.L. 2001. Effects of short-term treatment with corticotropin on the serum apolipoprotein pattern. Scand $J$ Clin Lab Invest 61:301-306.

5. Berg, A.L., Nilsson-Ehle, P., and Arnadottir, M. 1999. Beneficial effects of ACTH on the serum lipoprotein profile and glomerular function in patients with membranous nephropathy. Kidney Int 56:1534-1543.

6. Berg, A.L., and Nilsson-Ehle, P. 1996. ACTH lowers serum lipids in steroid-treated hyperlipemic patients with kidney disease. Kidney Int 50:538-542.

7. Arnadottir, M., Berg, A.L., Dallongeville, J., Fruchart, J.C., and Nilsson-Ehle, P. 1997. Adrenocorticotrophic hormone lowers serum Lp(a) and LDL cholesterol concentrations in hemodialysis patients. Kidney Int 52:1651-1655.

8. Xu, N., Ekstrom, U., and Nilsson-Ehle, P. 2001. ACTH decreases the expression and secretion of apolipoprotein B in HepG2 cell cultures. J Biol Chem 276:38680-38684.

9. Hussain, M.M. 2000. A proposed model for the assembly of chylomicrons. Atherosclerosis 148:1-15. 
10. Shelness, G.S., and Sellers, J.A. 2001. Very-low-density lipoprotein assembly and secretion. Curr Opin Lipidol 12:151-157.

11. Nilsson-Ehle, P., Carlstrom, S., and Belfrage, P. 1975. Rapid effect on lipoprotein lipase activity in adipose tissue of humans after carbohydrate and lipid intake. Scand J Clin Lab Invest 35:373-378.

12. Bitzen, U., Winqvist, M., Nilsson-Ehle, P., and Fex, G. 1994. Retinyl palmitate is a reproducible marker for chylomicron elimination from blood. Scand J Clin Lab Invest 54:611-613.

13. Nierenberg, D.W., and Lester, D.C. 1985. Determination of vitamins A and E in serum and plasma using a simplified clarification method and high-performance liquid chromatography. J Chromatogr 345:275-284.

14. Lovegrove, J.A., Isherwood, S.G., Jackson, K.G., Williams, C.M., and Gould, B.J. 1996. Quantitation of apolipoprotein B-48 in triacylglycerol-rich lipoproteins by a specific enzyme-linked immunosorbent assay. Biochim Biophys Acta 1301:221-229.

15. Peel, A.S., Zampelas, A., Williams, C.M., and Gould, B.J. 1993. A novel antiserum specific to apolipoprotein B-48: application in the investigation of postprandial lipidaemia in humans. Clin Sci (Lond) 85:521-524.

16. Hardarson, A., Sigurdsson, G., Olafsdottir, E., Dallongeville, J., Berg, A.L., and Arnadottir, M. 2001. Adrenocorticotrophic hormone exerts marked lipid-lowering effects in simvastatin-treated patients. J Intern Med 250:530-534.

17. Berg, A.L., Hansson, P., and Nilsson-Ehle, P. 1991. ACTH 1-24 decreases hepatic lipase activities and low density lipoprotein concentrations in healthy men. $J$ Intern Med 229:201-203.

18. Rubinstein, A., Gibson, J.C., Paterniti, J.R., Jr., Kakis, G., Little, A., Ginsberg, H.N., and Brown, W.V. 1985. Effect of heparin-induced lipolysis on the distribution of 
apolipoprotein e among lipoprotein subclasses. Studies with patients deficient in hepatic triglyceride lipase and lipoprotein lipase. J Clin Invest 75:710-721.

19. Connelly, P.W. 1999. The role of hepatic lipase in lipoprotein metabolism. Clin Chim Acta 286:243-255.

20. Hegele, R.A., Little, J.A., Vezina, C., Maguire, G.F., Tu, L., Wolever, T.S., Jenkins, D.J., and Connelly, P.W. 1993. Hepatic lipase deficiency. Clinical, biochemical, and molecular genetic characteristics. Arterioscler Thromb 13:720-728.

21. Hardman, D.A., Protter, A.A., Schilling, J.W., and Kane, J.P. 1987. Carboxyl terminal analysis of human B-48 protein confirms the novel mechanism proposed for chain termination. Biochem Biophys Res Commun 149:1214-1219.

22. Mehrabian, M., Sparkes, R.S., Mohandas, T., Klisak, I.J., Schumaker, V.N., Heinzmann, C., Zollman, S., Ma, Y.H., and Lusis, A.J. 1986. Human apolipoprotein B: chromosomal mapping and DNA polymorphisms of hepatic and intestinal species. Somat Cell Mol Genet 12:245-254.

23. Chen, S.H., Habib, G., Yang, C.Y., Gu, Z.W., Lee, B.R., Weng, S.A., Silberman, S.R., Cai, S.J., Deslypere, J.P., Rosseneu, M., et al. 1987. Apolipoprotein B-48 is the product of a messenger RNA with an organ-specific in-frame stop codon. Science 238:363-366.

24. Powell, L.M., Wallis, S.C., Pease, R.J., Edwards, Y.H., Knott, T.J., and Scott, J. 1987. A novel form of tissue-specific RNA processing produces apolipoprotein-B48 in intestine. Cell 50:831-840.

25. Davidson, N.O., and Shelness, G.S. 2000. APOLIPOPROTEIN B: mRNA editing, lipoprotein assembly, and presecretory degradation. Annu Rev Nutr 20:169-193.

26. Tache, Y., Martinez, V., Million, M., and Wang, L. 2001. Stress and the gastrointestinal tract III. Stress-related alterations of gut motor function: role of brain 
corticotropin-releasing factor receptors. Am J Physiol Gastrointest Liver Physiol 280:G173-177.

27. Monnikes, H., Tebbe, J.J., Hildebrandt, M., Arck, P., Osmanoglou, E., Rose, M., Klapp, B., Wiedenmann, B., and Heymann-Monnikes, I. 2001. Role of stress in functional gastrointestinal disorders. Evidence for stress-induced alterations in gastrointestinal motility and sensitivity. Dig Dis 19:201-211.

28. Fukudo, S., Nomura, T., and Hongo, M. 1998. Impact of corticotropin-releasing hormone on gastrointestinal motility and adrenocorticotropic hormone in normal controls and patients with irritable bowel syndrome. Gut 42:845-849.

29. Sakr, S.W., Attia, N., Haourigui, M., Paul, J.L., Soni, T., Vacher, D., and GirardGloba, A. 1997. Fatty acid composition of an oral load affects chylomicron size in human subjects. Br J Nutr 77:19-31.

30. Silva, K.D., Kelly, C.N., Jones, A.E., Smith, R.D., Wootton, S.A., Miller, G.J., and Williams, C.M. 2003. Chylomicron particle size and number, factor VII activation and dietary monounsaturated fatty acids. Atherosclerosis 166:73-84.

31. Wang, A.B., Liu, D.P., and Liang, C.C. 2003. Regulation of human apolipoprotein B gene expression at multiple levels. Exp Cell Res 290:1-12.

32. Anant, S., and Davidson, N.O. 2002. Identification and regulation of protein components of the apolipoprotein B mRNA editing enzyme. A complex event. Trends Cardiovasc Med 12:311-317.

33. Teng, B., Verp, M., Salomon, J., and Davidson, N.O. 1990. Apolipoprotein B messenger RNA editing is developmentally regulated and widely expressed in human tissues. J Biol Chem 265:20616-20620. 
34. Srivastava, R.A., Tang, J., Baumann, D., and Schonfeld, G. 1992. Hormonal and nutritional stimuli modulate apolipoprotein B mRNA editing in mouse liver. Biochem Biophys Res Commun 188:135-141.

35. Lorentz, A., Plonne, D., Schulze, H.P., and Dargel, R. 1996. Dexamethasone enhanced by insulin, but not by thyroid hormones stimulates apolipoprotein B mRNA editing in cultured rat hepatocytes depending on the developmental stage. FEBS Lett 391:57-60.

36. Thorngate, F.E., Raghow, R., Wilcox, H.G., Werner, C.S., Heimberg, M., and Elam, M.B. 1994. Insulin promotes the biosynthesis and secretion of apolipoprotein B-48 by altering apolipoprotein B mRNA editing. Proc Natl Acad Sci U S A 91:5392-5396.

37. Sjoberg, A., Oscarsson, J., Bostrom, K., Innerarity, T.L., Eden, S., and Olofsson, S.O. 1992. Effects of growth hormone on apolipoprotein-B (apoB) messenger ribonucleic acid editing, and apoB 48 and apoB 100 synthesis and secretion in the rat liver. Endocrinology 130:3356-3364. 


\section{Table 1}

Cortisol and glucose concentrations in the fasting state after four days' administration of ACTH and betamethasone.

\begin{tabular}{lcccc}
\hline & basal I & ACTH & basal II & betamethasone \\
\hline P-Cortisol $n m o l / L$ & $442(351-730)$ & $828(380-1312)$ & $414(338-576)$ & $11(8-19)^{* *}$ \\
P-Glucose $\mathrm{mmol} / \mathrm{L}$ & $4.9(4.5-5.3)$ & $5.4(5.0-6.1)^{*}$ & $4.9(4.5-5.1)$ & $5.3(5.2-5.6)^{*}$ \\
\hline
\end{tabular}

Volunteers underwent both ACTH $(n=10)$ and betamethasone $(n=8)$ administration with a 6month interval. Data are given as median (interquartile interval). $* P<0,05,{ }^{* *} P<0,01$ compared to the corresponding basal value. 


\section{Table 2}

Lipid and apolipoprotein concentrations in the fasting state after four days' administration of ACTH and betamethasone.

\begin{tabular}{|c|c|c|c|c|}
\hline & basal I & ACTH & basal II & betamethasone \\
\hline P-Cholesterol $\mathrm{mmol} / \mathrm{L}$ & $4.7(4.4-5.5)$ & $4.1(3.7-4.6)^{* *}$ & 4.6(3.9-5.1) & $4.9(4.5-5.5)$ \\
\hline P-Triglyceride $\mathrm{mmol} / \mathrm{L}$ & $0.95(0.60-1.45)$ & $0.85(0.65-1.30)$ & $0.99(0.66-1.25)$ & $1.05(0.80-1.30)$ \\
\hline S-Phospholipids $\mathrm{mmol} / \mathrm{L}$ & $3.02(2.42-3.22)$ & $2.51(2.25-2.69) * *$ & $2.90(2.47-3.25)$ & $2.90(2.83-3.16)$ \\
\hline P-HDL cholesterol $\mathrm{mmol} / \mathrm{L}$ & $1.62(1.36-2.08)$ & $1.57(1.44-1.88)$ & $1.73(1.49-1.99)$ & $1.94(1.74-2.28)^{*}$ \\
\hline P-ApoA-I $g / L$ & $1.28(1.13-1.61)^{\mathrm{B}}$ & $1.21(1.02-1.38)^{*}$ & $1.57(1.39-1.86)^{\mathrm{B}}$ & $1.60(1.47-1.77)$ \\
\hline P-LDL cholesterol $\mathrm{mmol} / \mathrm{L}$ & $2.97(2.35-3.55)$ & $2.23(1.75-2.62)^{* *}$ & $3.00(2.29-3.36)$ & $2.96(2.63-3.55)$ \\
\hline P-ApoB $g / L$ & $0.90(0.77-1.03)^{\mathrm{B}}$ & $0.75(0.68-0.89)^{* *}$ & $0.84(0.64-0.93)^{\mathrm{B}}$ & $0.90(0.78-1.02)$ \\
\hline P-ApoB-48 mg/L & $0.13(0.10-0.19)$ & $0.19(0.09-0.26)^{*}$ & NA & $0.20(0.13-0.26)^{* A}$ \\
\hline
\end{tabular}

Volunteers underwent both ACTH $(\mathrm{n}=10)$ and betamethasone $(\mathrm{n}=8)$ administration with a 6month interval. Data are given as median (interquartile interval). * $P<0,05$, ** $P<0,01$ compared to the corresponding basal value. ${ }^{\mathrm{A}} \mathrm{P}<0,05$ compared to basal I value. ${ }^{\mathrm{B}}$ The slight increase in P-ApoA-I and decrease in P-ApoB at basal II compared to basal I was due to changes in routine laboratory methods. 


\section{Legend to Figures}

\section{Figure 1}

a. Postprandial P-ApoB-48 levels after four days' administration of ACTH [open squares $\square$, $\mathrm{n}=8$ ], betamethasone [open circles $\bigcirc, \mathrm{n}=8$ ] and no drug administration [filled circles $\bullet, \mathrm{n}=8$ ]. Data represent mean of values. b. The accumulated change in P-ApoB-48 during the early postprandial phase (0-4 hours), expressed as AUC after ACTH, betamethasone and no drug administration. Data represent mean of individual areas \pm SEM.

\section{Figure 2}

Postprandial P-Triglycerides levels after four days' administration of ACTH [open squares $\square$, n=8], betamethasone [open circles $O, n=8$ ] and no drug administration [filled circles $\mathrm{n}=8]$. Data represent mean of values.

\section{Figure 3}

Postprandial S-Retinyl palmitate levels after four days' administration of ACTH [open squares $\square, n=8$ ], betamethasone [open circles $O, n=8$ ] and no drug administration [filled circles $\bullet, n=8]$. Data represent mean of values. 
Figure 1
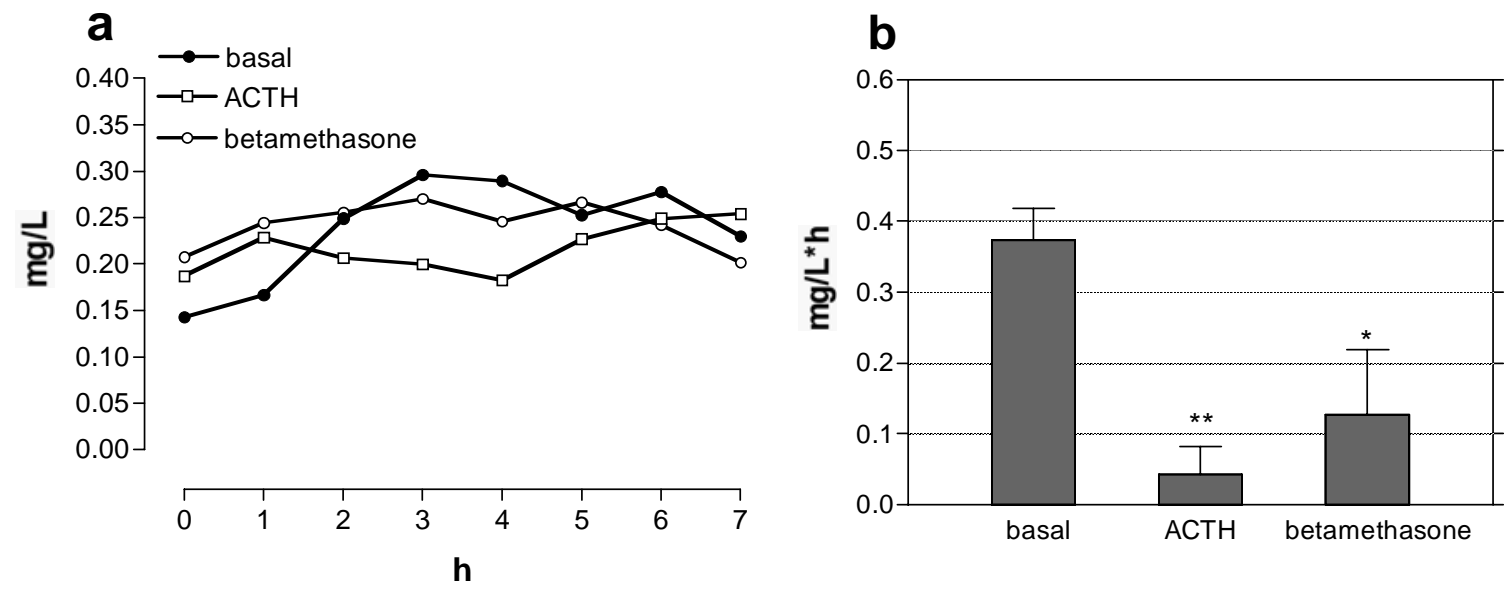
Figure 2

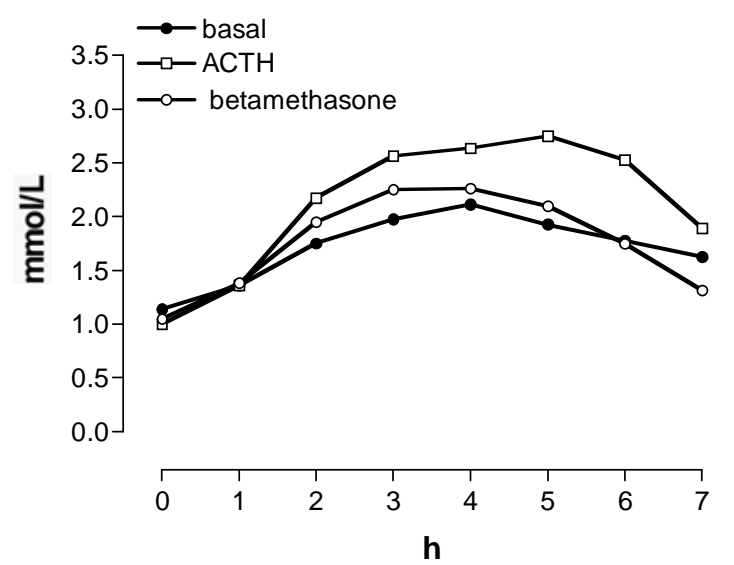


Figure 3

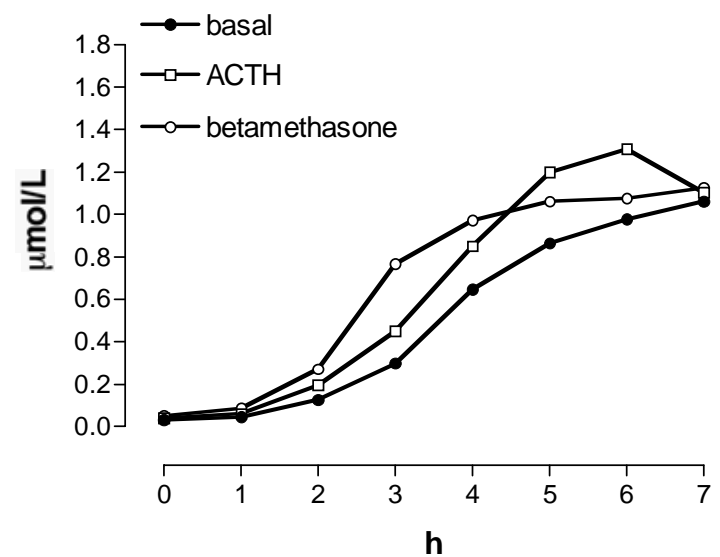

